\title{
2,4,6-TRIMETHYLPYRIDINIUM CHLOROCHROMATE (TMPCC) AS AN EFFICIENT AND NOVEL OXIDIZING AGENT FOR THE CHEMOSELECTIVE OXIDATION OF ALCOHOLS TO THE CORRESPONDING CARBONYL COMPOUNDS
}

\author{
LOTFI SHIRI*, ARASH GHORBANI-CHOGHAMARANI* \\ Department of Chemistry, Faculty of Science, Ilam University, P.O. Box 69315516, Ilam, Iran \\ (Received: September 22, 2008 - Accepted: December 30, 2008)
}

\begin{abstract}
A simple and efficient chemoselective oxidation of alcohols to the corresponding aldehydes and ketones using 2,4,6-trimethylpyridinium chlorochromate (TMPCC) in acetonitrile was described. A good range of primary and secondary benzylic alcohols was selectively oxidized under reflux conditions in good to excellent yields.
\end{abstract}

Keywords Alcohols, Oxidation, Heterogeneous, 2,4,6-Trimethylpyridinium chlorochromate (TMPCC).

\section{INTRODUCTION}

Oxidation is a fundamental transformation in organic synthesis and there are numerous methods reported in the literature ${ }^{1}$. The oxidation of alcohols to carbonyl compounds is one of the most important reactions in synthetic organic chemistry, and it is usually achieved using harmful different reagents. ${ }^{2}$ The conversion of primary and secondary alcohols to the corresponding carbonyl compounds is essential for the preparation of fragrances and food additives as well as of many synthetic intermediates in organic chemistry. ${ }^{3}$ Hence, search for effective systems that use environmentally benign and inexpensive oxidants to convert alcohols to carbonyl compounds remains an important challenge. Although a variety of reagents are capable of effecting these oxidations, ${ }^{4-}$ ${ }^{27}$ this transformation is a tricky step because the corresponding aldehydes from primary alcohols are very sensitive to the oxidizing agents and reaction conditions, being oxidized in a second step to the corresponding carboxylic acid compounds.

\section{RESULT AND DISCUSSION}

Consequently, we decided to choose a new reagent to overcome the above limitations. In addition, for our purpose both clean and easy work-up were also important. Continuing with our studies on the application of heterogeneous oxidizing systems $\mathrm{s}^{28-35}$ we decided to introduce TMPCC I as a new oxidizing reagent. It is interesting to note that preparing TMPCC is an easy and clean, without difficult work-up, procedure and pure reagent can be obtained only by crystallization in water (Scheme 1)<smiles>Cc1cc(C)nc(C)c1</smiles>

THPCC

Additionally, there is current general research interest in heterogeneous systems $\mathrm{s}^{36-42}$ due to the importance such systems have in industry and developing technologies. We report here a simple, cheap and chemoselective method for the effective oxidation of primary and secondary benzylic alcohols to their corresponding carbonyl compounds=using TMPCC under reflux conditions in acetonitrile.

Initially, we screened different solvents for the oxidation of 2-chlorobenzyl alcohol and found that acetonitrile is the best solvent in terms of activity and selectivity (Table 1).
Table 1. Oxidation of 2-chlorobenzyl alcohol using TMPCC in different solvents under reflux conditions. ${ }^{\mathrm{a}}$

\begin{tabular}{|c|c|c|c|}
\hline Entry & Solvent & Time (Min) & Yield (\%) $^{\mathbf{b}}$ \\
\hline 1 & Acetonitrile & 100 & 100 \\
\hline 2 & Acetone & 120 & 35 \\
\hline 3 & Chloroform & 120 & 40 \\
\hline 4 & Dichloromethane & 120 & 10 \\
\hline 5 & Tetrahydrofurane & 120 & 15 \\
\hline 6 & Diethyl ether & 120 & 0 \\
\hline
\end{tabular}

a 2-chlorobenzyl alcohol : TMPCC $=1 \mathrm{mmol}: 2 \mathrm{mmol}$. ${ }^{\mathrm{b}}$ Yield was determined by 2,4-dinitrophenyl hydrazone derivatives.

Different types of primary and secondary benzylic alcohols $\mathbf{1}$ were subjected to the oxidation reaction in the presence of TMPCC in acetonitrile (Scheme 2) the results for this transformation are summarized in Table 2.<smiles></smiles>

$\mathrm{R}^{1}, \mathrm{R}^{2}$ : Aryl, Vinyl or $\mathrm{H}$

Scheme 2 
Table 2. Oxidation of primary and secondary benzylic alcohols $\mathbf{1}$ to their corresponding carbonyl compounds $\mathbf{2}$ with TMPCC under reflux conditions in acetonitrile.

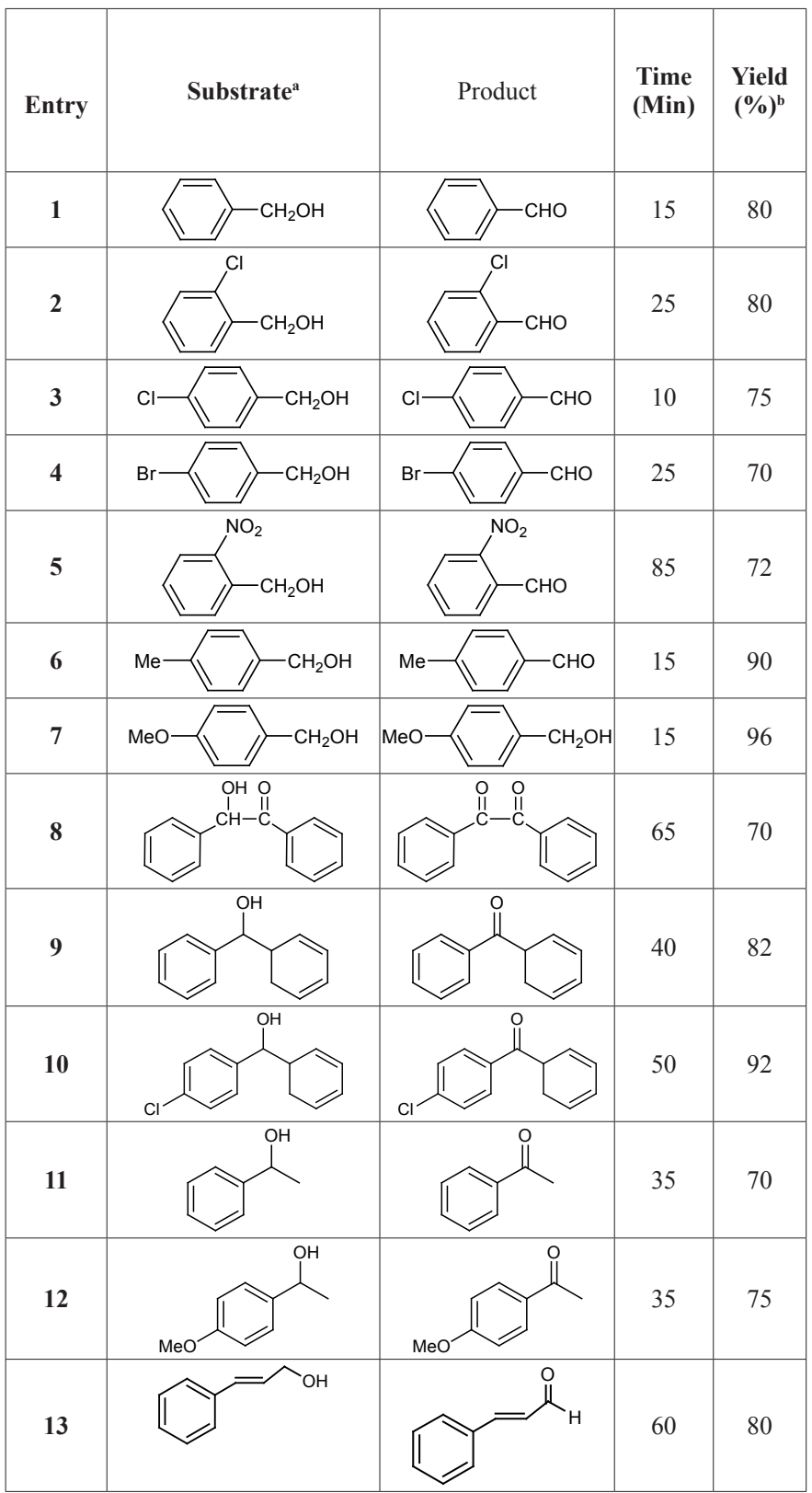

${ }^{\mathrm{a}}$ substrate : TMPCC $(1: 2 \mathrm{mmol}),{ }^{\mathrm{b}}$ Isolated yields.

To show the chemoselectivity of the described system cinnamyl alcohol was converted to cinnamaldehyde in good yields and carbon-carbon double bond remains unchanged in the course of reaction (Table 2, entry 13).

It is of interest that, this oxidizing system chemoselectively oxidized all primary benzylic alcohols to the corresponding aldehydes without over oxidation (Table 2, entries 1-7, Scheme 3).

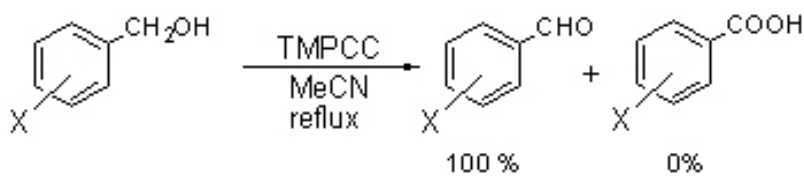

Scheme 3
To show the efficiency of the described system in comparison with previously reported chromium-containing oxidants in the literature, we compared our obtained results for the oxidation of 4-methoxybenzyl alcohol (as a typical example) with the best of the well-known data from the literature as shown in the Table 3.

Table 3. Efficiency comparison of various chromium-containing oxidizing systems for the oxidation of 4-methoxybenzyl alcohol

\begin{tabular}{|c|c|c|c|c|}
\hline Entry & Reagent & Time (Min) & $\begin{array}{c}\text { Yield } \\
(\%)^{\mathbf{a}}\end{array}$ & Reference \\
\hline 1 & $\mathrm{TMPCC}^{2}$ & 15 & 96 & This work \\
\hline 2 & $\mathrm{SiO}_{2}-\mathrm{CrO}_{2}-\mathrm{SiO}_{2}$ & 10 & 90 & {$[9]$} \\
\hline 3 & $\mathrm{Ph}_{3} \mathrm{PMeClCrO}_{3}$ & 30 & 90 & {$[24]$} \\
\hline 4 & $\mathrm{PCC}_{2} \mathrm{Al}_{2} \mathrm{O}_{3}$ & 60 & 82 & {$[43]$} \\
\hline 5 & $\left(\mathrm{NH}_{4}\right)_{2} \mathrm{Cr}_{2} \mathrm{O}_{7}$ & 25 & 82 & {$[44]$} \\
\hline 6 & $\mathrm{CrO}_{3} / \mathrm{H}_{5} \mathrm{IO}_{6}$ & 60 & 90 & {$[45]$} \\
\hline
\end{tabular}

${ }^{a}$ Isolated yield.

We hoped that the TMPCC would be an excellent oxidizing reagent for general oxidation reactions.

\section{EXPERIMENTAL}

Chemicals were purchased from Fluka, Merck and Aldrich chemical companies. The oxidation products were characterized by comparison of their spectral (IR, ${ }^{1} \mathrm{H}$ NMR, and ${ }^{13} \mathrm{C}$ NMR) and physical data with authentic samples.

\section{Preparation of 2,4,6-trimethylpyridinium chlorochromate}

A $100 \mathrm{~mL}$ round bottom flask was equipped with a constant-pressure dropping funnel containing 2,4,6-trimethylpyridine $(13.4 \mathrm{~mL}, 0.1 \mathrm{~mol})$. The flask was charged with $\mathrm{CrO}_{3}(10 \mathrm{~g}, 0.1 \mathrm{~mol})$ and $\mathrm{HCl} 6 \mathrm{M}(18.4 \mathrm{~mL})$. This mixture was cooled down to $0{ }^{\circ} \mathrm{C}$ then 2,4,6-trimethylpyridine was added dropwise for $10 \mathrm{~min}$ at room temperature. After the addition, the mixture was shaken for 15 min then kept in the freezer for $24 \mathrm{~h}$. Orange jelly sediment was obtained. The jelly reagent was discolored with active charcoal and crystallized in water then dried by oven. 2,4,6-Trimethylpyridinium chlorochromate as orange solid was obtained quantitatively.

Oxidation of benzyl alcohol to the benzaldehyde: as a typical procedure

A suspension of benzyl alcohol $(0.108 \mathrm{~g}, 1 \mathrm{mmol})$ and 2,4,6trimethylpyridinium chlorochromate $(0.515 \mathrm{~g}, 2 \mathrm{mmol})$ in $\mathrm{MeCN}(15 \mathrm{~mL})$ was stirred under reflux conditions for $15 \mathrm{~min}$ (the progress of the reaction was monitored by TLC). After reaction completion, reaction mixture was cooled to room temperature then filtered off. The residue was washed with diethyl ether $(3 \times 10 \mathrm{~mL})$ then solvent was removed using rotary evaporation. The product was purified by short column chromatography using diethyl ether as eluent. Benzaldehyde was obtained in $80 \%$ yield $(0.085 \mathrm{~g})$.

\section{CONCLUSION}

In summary, the availability of the reagents, easy and clean work-up, good conversion of alcohols in high yields make this an attractive methodology. We believe that the present methodology could be an important addition to those methods already existing.

\section{ACKNOWLEDGEMENT}

Financial support for this work by the research affair, Ilam University, Ilam, Iran, is gratefully acknowledged.

\section{REFERENCES}

1. M. Hudlicky, ACS Mongraph, 186 (1990).

2. S.V. Ley, A. Madin, B.M. Trost, I. Fleming (Eds.), Comprehensive Organic Synthesis, vol. 7, Pergamon Press, Oxford, 1991, p. 251.

3. R.A. Sheldon, I.W.C.E. Arends, A. Dijksman, Catal. Today, 57, 157, (2000).

4. A. Miyata, M. Murakami, R. Irie, T. Katsuki, Tetrahedron Lett., 42, 7067, (2001). 
5. (a) M.A. Zolfigol, M. Torabi, S.E. Mallakpour, Tetrahedron, 57, 8381, (2001); (b) F. Shirini, M.A. Zolfigol, S.E. Mallakpour, A.R. Hajipour, I. Baltork, Tetrahedron Lett., 43, 1555, (2002).

6. M.A. Zolfigol, Tetrahedron, 57, 9509, (2001).

7. M.A. Zolfigol, F. Shirini, A. Ghorbani-Choghamarani, I. MohammadpoorBaltork, Green Chem., 562, (2002).

8. K. Fujita, S. Furukawa, R. Yamaguchi, J. Organomet. Chem., 649, 289, (2002).

9. A.R. Hajipour, L. Khazdooz, A.E. Ruoho, J. Iran. Chem. Soc., 2, 315, (2005).

10. M. Moghadam, S. Tangestaninejad, V. Mirkhani, B. Karami, N. Rashidi, H. Ahmadi, J. Iran. Chem. Soc., 3, 64, (2006).

11. K. Hara, S. Tayama, H. Kano, T. Masuda, S. Takakusagi, T. Kondo, K. Uosaki, M. Sawamura, Chem. Commun., 4280, (2007).

12. B. Karimi, A. Biglari, J.H. Clark, V. Budarin, Angew. Chem. Int. Edit., 46, 7210, (2007).

13. Y. Luan, G. Wang, R.L. Luck, M. Yang, X. Han, Chem. Lett., 36, 1236, (2007).

14. R. Ghorbani-Vaghei, M. Amiri, M. Chegny, H. Veisi, S. Afr. J. Chem-SAfr. T., 60, 58, (2007).

15. P. Lahtinen, J.U. Ahmad, E. Lankinen, P. Pihko, M. Leskela, T. Repo, J. Mol. Catal. A-Chem., 275, 228, (2007).

16. Z. Opre, D. Ferri, F. Krumeich, T. Mallat, A. Baiker, J. Catal., 241, 287, (2006).

17. M. Yang, K.-T. Yip, J.-H. Pan, Y.-C. Chen, N.-Y. Zhu, D. Yang, Synlett, 3057, (2006).

18. J.M. Grill, J.W. Ogle, S.A. Miller, J. Org. Chem., 71, 9291, (2006).

19. R. Ozen, N.S. Kus, Monatsh. Chem., 137, 1597, (2006).

20. M.S. Yusubov, K.-W. Chi, J.Y. Park, R. Karimov, V.V. Zhdankin, Tetrahedron Lett. 47, 6305, (2006).

21. M.A. Zolfigol, F. Shirini, A. Ghorbani-Choghamarani, Synthesis, 2043, (2006).

22. M.M. Heravi, N. Farhangi, K. Bakhtiari, M. Ghassemzadeh, J. Chin. Chem. Soc., 53, 643, (2006).

23. M. Lei, R.-J. Hu, Y.-G. Wang, Tetrahedron, 62, 8928, (2006).

24. M.A. Zolfigol, F. Shirini, I. Mohammadpoor-Baltork, A. GhorbaniChoghamarani, M. Hajjami, M. Sedaghat, Mendeleev Commun., 113, (2005).
25. N. Feizi, H. Hassani, M. Hakimi, B. Kor. Chem. Soc., 26, 2084, (2005).

26. V.V. Potekhin, S.N. Soloveva, V.M. Potekhin, Russ. Chem. B., 52, 2668 (2003).

27. L.Z. Wang, J.D. Lou, L.Y. Zhu, Oxid. Commun., 27, 906, (2004).

28. M.A. Zolfigol, M. Bagherzadeh, K. Niknam, F. Shirini, I. MohammadpoorBaltork, A. Ghorbani-Choghamarani, M. Baghbanzadeh, J. Iran. Chem. Soc. 3, 73, (2006).

29. M.A. Zolfigol, M. Bagherzadeh, S. Mallakpour, G. Chehardoli, E. Kolvari, A. Ghorbani-Choghamarani, N. Koukabi, Catal. Commun. 8, 256, (2007).

30. M.A. Zolfigol, M. Bagherzadeh, S. Mallakpour, G. Chehardoli, A. Ghorbani-Choghamarani, N. Koukabi, M. Dehghanian, M. Doroudgar, J. Mol. Catal. A-Chem. 270, 219, (2007).

31. M.A. Zolfigol, F. Shirini, G. Chehardoli, E. Kolvari, J. Mol. Catal. A-Chem. 265, 272, (2007).

32. K. Niknam, M.A. Zolfigol, T. Sadabadi, J. Iran. Chem. Soc., 4, 199 , (2007).

33. K. Niknam, M.A. Zolfigol, J. Iran. Chem. Soc., 3, 59, (2006).

34. A. Bamoniri, M.A. Zolfigol, I. Mohammadpoor-Baltork, B.F. Mirjalili, J Iran. Chem. Soc., 3, 85, (2006).

35. E. Kolvari, A. Ghorbani-Choghamarani, P. Salehi, F. Shirini, M.A. Zolfigol, J. Iran. Chem. Soc. 4, 126, (2007).

36. M.A. Zolfigol, F. Shirini, A. Ghorbani-Choghamarani, A. Taqian-Nasab, H. Keypour, S. Salehzadeh, J. Chem. Res-S, 420, (2000).

37. M.A. Zolfigol, A. Bamoniri, Synlett, 1621, (2002).

38. M.A. Zolfigol, Molecules, 6, 694, (2001).

39. M.A. Zolfigol, M. Kiany-Borazjani, M.M. Sadeghi, I. MohammadpoorBaltork, H.R. Memarian, Synth. Commun., 30, 551, (2000).

40. M.M. Sadeghi, I. Mohammadpoor-Baltork, H.R. Memarian, S. Sobhani, Synth. Commun., 30, 1661, (2000).

41. M.A. Zolfigol, M.H. Zebarjadian, M.M. Sadeghi, I. MohammadpoorBaltork, H.R. Memarian, M. Shamsipur, Synth.Commun., 32, 2803, (2002).

42. M.A. Zolfigol, E. Ghaemi, E. Madrakian, Synlett, 191, (2003).

43. S. Bhar, S.K. Chaudhuri, Tetrahedron, 59, 3493, (2003).

44. F. Shirini, M.A. Zolfigol, M. Khaleghi, Bull. Korean Chem. Soc., 24, 1021 (2003).

45. S. Zhang, L. Xu, M.L. Trudell, Synthesis, 1757, (2005). 